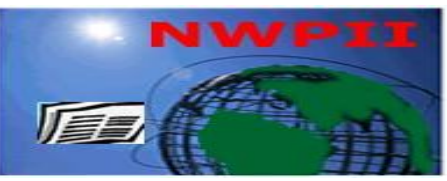

American Journal of Biomedical Sciences

ISSN: 1937-9080

nwpii.com/ajbms

\title{
Research and Design a Non- Invasive Blood Glucose Measuring Module
}

\section{Duong Trong Luong*, Nguyen Xuan Huy, Dao Viet Hung, Nguyen Thai Ha, Nguyen Duc Thuan}

Department of Electronic Technology and Biomedical Engineering, Hanoi University of Science and Technology,

Vietnam

*Corresponding Author

Duong Trong Luong

Department of Electronic Technology and Biomedical Engineering

Hanoi University of Science and Technology

Vietnam

Email: luong.duongtrong@hust.edu.vn

Received:10 May 2018; | Revised:25 May 2018; | Accepted: 30 August 2018

\section{Abstract}

One of the best challenges in healthy field in 21th century is diabetes because the number of patients is rising faster and faster. Prevention of complications of diabetes, we have to continuously monitor blood glucose level. In this paper, we introduce our research about non- invasive blood glucose measuring device which used infrared light $1550 \mathrm{~nm}$. Our goals are developing the non- invasive glucose measuring device, it is more convenient in measuring and following glucose index in patient's body. We can follow this index continuously, without pain and slow down problems about infectious diseases. Beside it, when using our device, the price is decreased because it does not use consumable supplies. But the accuracy of the measuring is still not high. We tested this device in living body of 2 volunteers in 10 days then compared the results of our device with those of invasive device used in the market, our device gives correlation coefficient from 0.870 to 0.995 .

Keywords: Non-invasive, Infrared light, Blood glucose,Diabetes

\section{Introduction}

In Viet Nam, the prevalence of diabetes is growing at alarming rates and has almost doubled within the past 10 years. Currently, it's estimated that one in every 20 Vietnamese adults has diabetes. In addition, the number of people with a prediabetic condition is three times higher than those with diabetes [1]. International Diabetes Federation (IDF) published a survey, which said in Vietnam more than $50 \%$ diabetes patients under 60 years old died every year. Patients in poor regions have many troubles in glucose testing. They almost use traditional invasive method (finger-prick) at medical organization. Some of them buy mobile device to check glucose level by themselves but it is complicated and easy to infect diseases. Therefore, researching and developing a non- invasive device which is convenient, fast and cheap have many advances in treatment and diagnostic diabetic 
diseases. This device is not only increased quality in screening test but also can able to use like personal equipment in all family thus higher convenient in diabetic diseases testing level. Up to now, there are many researches on non- invasive glucose measuring which published such as: Mid-infrared spectroscopy ${ }^{[3]}$, Near infrared spectroscopy ${ }^{[4]}[5]$, Raman spectroscopy ${ }^{[6][7]}$, Ultrasound technology ${ }^{[8]}$ [9].

However, all of them have some disadvantages which need to improve.

Mid-infrared spectroscopy (MIR) method of Vonlilienfeldtoal $\mathrm{H}$ el al used principle same to infrared spectroscopy. In other words, it is the absorption measurement of MIR frequency by a sample positioned in the path of an MIR beam. It is based on light in the $2500-25000 \mathrm{~nm}$ region of the spectrum. Absorption differences when MIR light meets human tissues can be represented by certain modeling techniques in spectral quantitative analysis. A partial least squares algorithm is now normally used for multivariate calibration for these constituents. MIR exhibits decreased scattering phenomena, yet increased absorption, because of the higher wavelengths compared with near infrared (NIR) spectroscopy ${ }^{[3]}$. Light can penetrate skin to a depth of a few micrometers. As a result, only reflected light can be considered, because there is no light transmitted through a body segment. Moreover, another possible advantage of MIR spectroscopy is that the response peaks of glucose and other compounds are sharper with MIR than with NIR, where they are often broad and weak. Limitations Poor penetration is the main limitation of MIR. Other limitations, as with NIR, include problems with confounding factors, such as water content in blood. Near infrared (NIR) spectroscopy of Heise $\mathrm{HM}$ el al is located in the wavelength region of 730-2500 $\mathrm{nm}$. The principle is similar to that of MIR spectroscopy. NIR spectra are made up of broad bands corresponding to overlapping peaks: the overtones (ie, first, second, third, and combination overtones), formed by molecular vibrations. It allows blood glucose measurement in tissues by variations of light intensity, generates one of the weakest NIR absorption signals per concentration unit of the body's major components. Maruo et al demonstrated the efficacy of this approach in vivo, using NIR diffuse reflectance spectroscopy through fiber optics on diabetes patients' forearms. The results showed positive signs on the correlation between predicted values and the reference glucose levels. Arnold and Small also reported that, although measurement errors of NIR spectroscopy are too large for clinical purposes, these experimental results demonstrate the possibility of noninvasive blood glucose measurements based on transmittance and reflectance. Heise et al, one of the pioneers in noninvasive blood glucose monitoring, has published much on NIR techniques [4][5]. Raghavachari reported that glucose generates one of the weakest NIR absorption signals per concentration unit of the body's major components ${ }^{[6]}$. Maruo et al demonstrated the efficacy of this approach in vivo, using NIR diffuse reflectance spectroscopy through fiber optics on diabetes patients' forearms ${ }^{[7]}$. The results showed positive signs on the correlation between predicted values and the reference glucose levels. Arnold and Small also reported that, although measurement errors of NIR spectroscopy are too large for clinical purposes, these experimental results demonstrate the possibility of noninvasive blood glucose measurements ${ }^{[8]}$. Advantages of this method is the high sensitivity of the photoconductive detectors is the main advantage of NIR spectroscopy. Water is reasonably transparent to the signal bandwidth used by NIR, which makes it possible to use for blood glucose monitoring. In addition, the measuring signal has high energy compared with MIR spectroscopy. Perhaps even more important, this method is less expensive than MIR. Materials are relatively low in cost, and there is a wide range of commercial products available. These advantages make NIR popular in this research area. Despite much promising work, researchers still cannot overcome important shortcomings, in particular, the scanning pressure that must be applied, physiological differences not related to blood glucose, the relatively small fraction of glucose in blood, weak correlation, and hardware sensitivity and stability.

Raman spectroscopy of Berger AJ anfdel al is based on the use of a laser light to induce oscillation and rotation in human fluids containing glucose. Because the emission of scattered light is influenced 
by molecular vibration, it is possible to estimate glucose concentration in human fluids ${ }^{[9]}$. This effect depends on the concentration of the glucose molecules. This technique can measure very weak signals, even in human fluids. The wavelength range of Raman spectrum is considered to be 200 $\mathrm{cm}-1$ to $2,000 \mathrm{~cm}-1^{[10]}$. Raman spectrum of glucose can be differentiated from those of other compounds in this band.

Raman spectroscopy usually provides sharper and less overlapped spectra compared to NIR spectroscopy. The intensity of spectral features is proportional to the concentration of the particular species, and the spectra are less sensitive to temperature changes. Moreover, it is comparatively less sensitive to water, and the interference from luminescence and fluorescence phenomena is only modest. The main limitations are related to instability of the laser wavelength and intensity, and long spectral acquisition times. In addition, as the power of the light source must be kept low to prevent injury, the signal-to-noise ratio is significantly reduced. Moreover, as with NIR spectroscopy, interference from other compounds remains a problem.

Ultrasound technology of Lee $\mathrm{S}$ and el al ${ }^{[11]}$ is based on low-frequency ultrasound, which penetrates the skin for blood glucose monitoring. While this approach has theoretical potential, it seems that no further works has been done since Lee's group reported their laboratory results on rat skin [11]. A variation, named photoacoustic spectroscopy, is being used, which is based on the use of a laser light for the excitation of a fluid and for measuring the resulting acoustic response ${ }^{[12]}$. The fluid is excited by a short laser pulse with a wavelength that is absorbed by a particular molecular species in the fluid. Light absorption causes microscopic localized heating in the medium, which generates an ultrasound pressure wave that is detected by a microphone. The principle of the photoacoustic method is that an energy source irradiates the skin surface, causing thermal expansion in the illuminated area. An acoustic wave releases because of the energy of the thermal expansion. The detection of glucose with this technique is based on measuring the changes of the peakto-peak value of the signal, which varies according to the glucose content of the blood.

In this paper, we present a method of design non- invasive blood glucose measure module based on principle of light absorption. This technology can provide higher sensitivity than traditional spectroscopy in the determination of glucose, because of the relatively better photoacoustic response of blood, as compared with water. This makes it easier to distinguish hydrocarbons and glucose $^{[11]}$. In addition, the laser light wavelengths that can be used have a wide range, from ultraviolet to NIR.

\section{Methodology}

\subsection{Infrared absorbance of skin tissues}

Most of soft tissues will allow both infrared light and visible light transmit it in a stable area. Cause selected absorption of blood in the hand, transmitted light is red ${ }^{[14]}$.

Non- invasive glucose measuring employ by analysis knit the finger transmitted light. Absorption of skin depends on blood ingredients such as water, hemoglobin, lipid and glucose. But absorption light of these blood ingredients depends more on wavelength of transmitted light. In each wavelength, the match between ingredients will change and absorb the light ${ }^{[14]}$. Concentration of glucose is able to determine by analysis the change of water in wavelength or intensity of transmitted light. Glucose in blood can be calculated when we measure infrared light which transmit through top of finger in many different wavelength (from $700 \mathrm{~nm}$ to $2500 \mathrm{~nm}$ ) as shown in Fig.1.

In Fig.1, we can see, absorption of glucose is 0.9 and absorption of water is less than 0.1 at wavelength $1550 \mathrm{~nm}$. Therefore, wavelength $1550 \mathrm{~nm}$ will decrease the effect of water when we determine glucose level in blood. At this wavelength, absorption of glucose is also higher therefore we are able to realize changed concentration of glucose in blood. Light absorption of skin, blood and water are stable in the finger, just glucose and blood color are unstable. But blood color effects trivially in infrared absorption of blood. 


\section{Glucose Water Spectra}

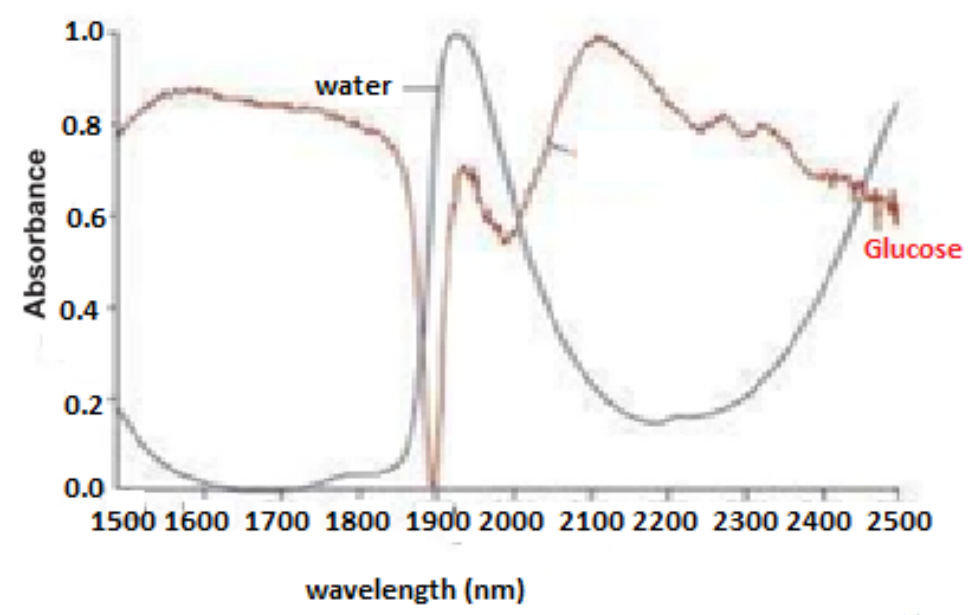

Figure 1: Absorption spectra of water and glucose ${ }^{[14]}$

\subsection{Principles}

Background of principle in blood glucose measuring is based on glucose in blood absorb infrared light more than the rest part of skin tissues. When glucose concentration increase, infrared light is higher absorbed therefore transmitted light intensity is lower. By measuring of light intensity transmit through the top of finger, we can be calculated glucose concentration in blood.

Lambert Beer theory

Beer-Lambert Law is the linear relationship between absorbance and concentration of an absorbing species. The general Beer-Lambert law is usually written as:

$$
A=\epsilon \times \ell \times c
$$

Where $\mathrm{A}=$ absorbance

$\epsilon=$ molar extinction coefficient,

$\ell=$ pathlength

$\mathrm{c}=$ concentration

Infrared light will expose from LED with suitable wavelength. The transmitted light is absorbed, reflected ad scattered by tissues and blood at the top of finger. By placing detector system at the opposite site of the LED, we can have the light which includes information about coefficients in the blood.

By reading the voltage values which is achieved before and after we put the finger on the system, we enable to calculate light intensity before and after absorption:

$$
\mathrm{P}=\frac{\mathrm{V}_{\mathrm{o}}}{\Re \times \mathrm{R}_{\mathrm{L}}}
$$

Application in Lambert beer theory, we have:

$$
A=-\log \frac{P}{P_{o}}
$$

The relationship of absorbance and concentration:

concentration: $\mathrm{c}=\frac{\mathrm{A}}{\epsilon \times \ell}$

where:

$\epsilon=$ molar extinction coefficient,

$\ell=$ pathlength or thickness of finger

$$
\mathrm{C}=\frac{-\log \frac{\mathrm{V}}{\mathrm{V}_{\mathrm{o}}}}{\epsilon \times \ell}
$$

The block diagram of designed non- invasive blood glucose measuring is shown in Figure 2.

We use IR LED have wavelength of $1550 \mathrm{~nm}$. We used IR photodiode which has achieved range from $700 \mathrm{~nm}$ to $1700 \mathrm{~nm}$. The most sensitive wavelength is $1550 \mathrm{~nm}$. IR LED and photodiode will be placed at the best suitable position so that the absorbance and scattering which we do not want to from skin and remaining component are smallest. Photon beam transmits through the top of finger and go to photodiode make a current. This current will be converted to voltage value by using current to voltage converter. Received signals can be noise by heartbeat, electromagnetic noise. Therefore, the 
suitable filter is low pass filter $0.4 \mathrm{~Hz}$ to eliminate these noises.

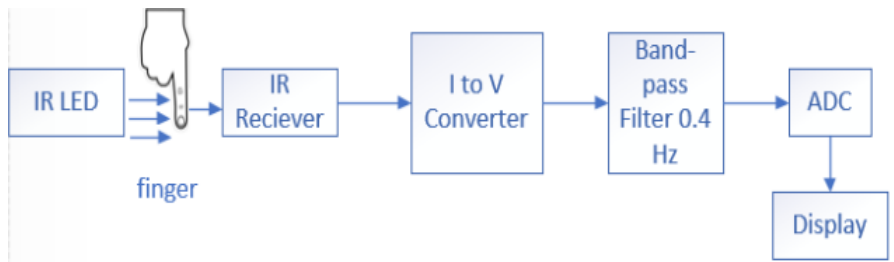

Figure 2: Block diagram of designed non- invasive blood glucose measuring

\subsection{Hardware implementation}

Sensor clip: we used the cover of finger clip SpO2 with IR LED and Photodiode are placed symmetrically at the both sides of clip.

Source block: we used battery which is powered more than $9 \mathrm{~V}$. This power will supply for source block using ICL 7660s. It will be created a symmetrically electric source $9 \mathrm{~V}$ which supplies for all of the system.

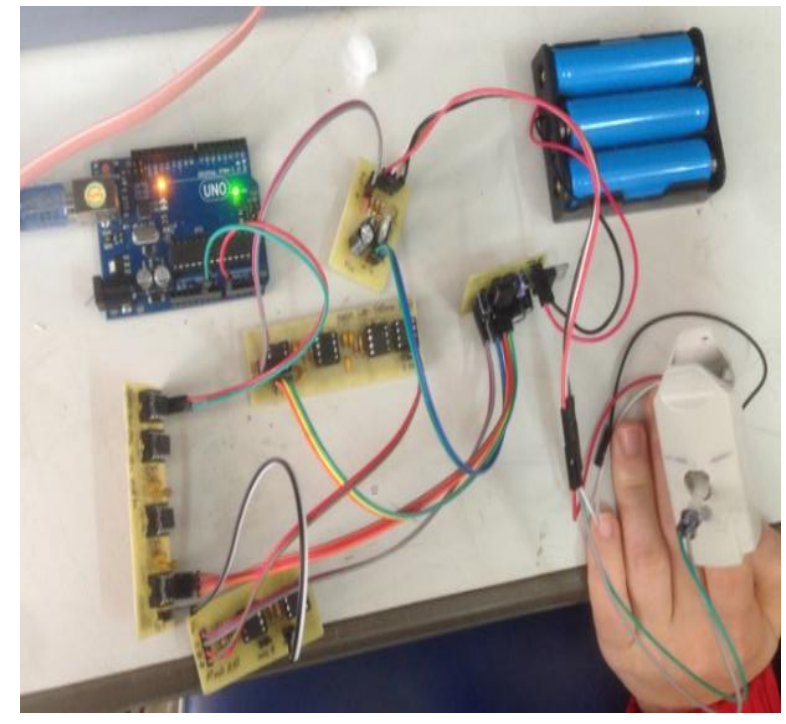

Figure 3: Design non- invasive blood glucose module

Receiver block: This block will supply power for IR LED and convert the received current signals from photodiode to voltage values.

First filter block: it is a low pass filer level 4 with cut off frequency is $0.4 \mathrm{~Hz}$. It helps eliminate all of the noise and AC signals which are unwanted from received signals.
Application block: There are 2 reasons to use this block. Firstly, received signals are very small. Secondly, resolution of ADC is not enough to process small signals. Therefore, we must use application block to process and make it bigger.

Second filter block: it is same to first filter block. It makes sure that only DC signals can pass through.

Processing ADC and display block: Arduino UNO R3 is employed. It has ADC block 10 bits and can be displayed directly on Serial Monitor.

\section{Results}

The Calibration of absorption and voltage.

Results from current output and blood glucose measuring on volunteers in different time such as before and after they have breakfast, we calculate absorbance of the finger which depends on voltage output. Therefore, we can find the equation to calculate the glucose concentration of the proposes device.

Results from the calibration experiments show that the output voltage of the sensor increases in direct proportion with increase of glucose concentration. The relationship between glucose concentration and voltage is linear as described in the equation:

$$
\epsilon \times l=0.1523 . \mathrm{V}-0.0061
$$

Equation (6) will be illustrated in Fig.4:

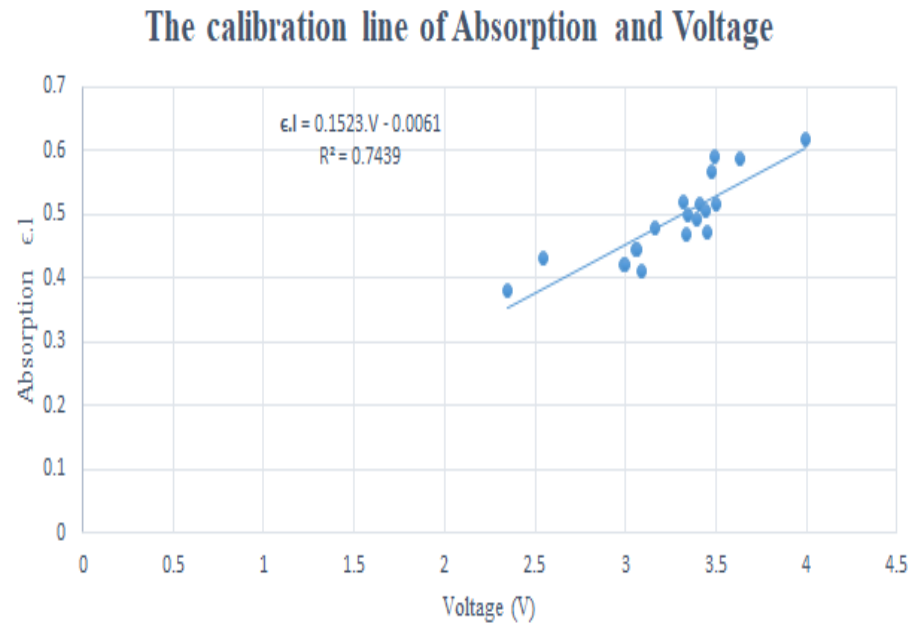

Figure 4: The Calibration of absorption and voltage 
We can measure the glucose concentration by using Lambert- Beer theory:

$$
\mathrm{C}=\frac{-\log \frac{\mathrm{V}}{\mathrm{V}_{\mathrm{o}}}}{0.1523 . \mathrm{V}-0.0061}
$$

We used both non- invasive blood glucose measuring which is proposed and invasive blood glucose device which are sold in the market (Safe
Accu company) to measure the glucose concentration on 2 volunteers before and after they have breakfast 2 hours. This process lasted continuously in 10 days. The result of the measuring is shown in table 1 .

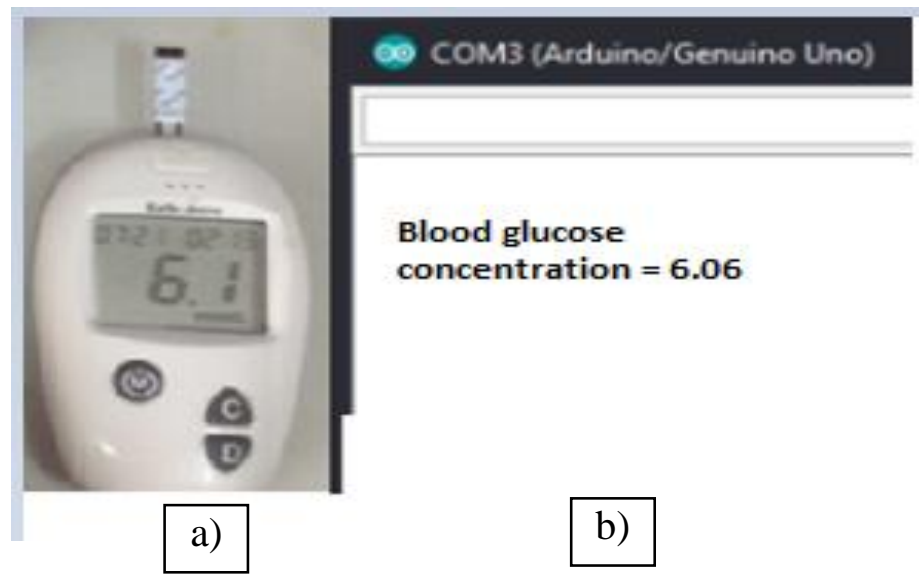

Figure 5: a) The glucose concentration is measured by the device in the maket; b) The glucose concentration is measured by proposed method

To evaluate the inaccuracy of the proposed method, we calculated the percent of inaccuracy after each measuring by the equation below:

$$
\% \text { Error }=\frac{\mid \text { Glucose }_{\text {invasive }}-\text { Glucose }_{\text {non-invasive }} \mid}{100 \%} \times
$$

The technology is sensitive to interference from some biological compounds, temperature fluctuations, and pressure changes. Moreover, when the laser light transverses a dense medium, the photoacoustic signal may be affected by scattering phenomena, which may possibly cause an adverse effect similar to that of NIR spectroscopy. Another disadvantage is that the instrumentation is expensive and sensitive to environmental parameters.
The best important reason makes the measuring inaccuracy is the different in the thickness of finger and cuticle. Addition to, there are some ambient factor effect on proposed module.

\section{Conclusion}

Based on the researches and results from experiments, the change of infrared absorbance of glucose at wavelength of $1550 \mathrm{~nm}$ is very strong. The proposed device can be applied in the real life. But the inaccuracy of the measuring is still quite high, we must to develop it about hardware implement to improve it accuracy. We can achieve more goals in non- invasive blood glucose measuring in the near future. 
Table 1: The comparation of glucose concentration between non- invasive and invasive methods

\begin{tabular}{|c|c|c|c|c|c|c|}
\hline \multirow{2}{*}{ Day } & \multicolumn{3}{|c|}{ Subject 1 } & \multicolumn{3}{c|}{ Subject 2 } \\
\cline { 2 - 7 } & $\begin{array}{c}\text { Safe } \\
\text { Accu }\end{array}$ & $\begin{array}{c}\text { Device } \\
\text { proposed }\end{array}$ & $\begin{array}{c}\% \\
\text { Error }\end{array}$ & $\begin{array}{c}\text { Safe } \\
\text { Accu }\end{array}$ & $\begin{array}{c}\text { Device } \\
\text { proposed }\end{array}$ & \% Error \\
\hline 1 & 6.9 & 6.45 & 6.5 & 6.1 & 5.68 & 6.9 \\
\hline 2 & 4.5 & 4.60 & 2.29 & 6.9 & 6.44 & 6.64 \\
\hline 3 & 5.5 & 5.38 & 2.25 & 5.8 & 5.58 & 3.87 \\
\hline 4 & 6 & 6.04 & 0.64 & 5.5 & 5.54 & 0.69 \\
\hline 5 & 6.5 & 6.30 & 3.11 & 7 & 6.22 & 11.19 \\
\hline 6 & 6 & 5.46 & 9.07 & 6.5 & 6.27 & 3.47 \\
\hline 7 & 5.7 & 5.67 & 0.52 & 4.8 & 5.14 & 7.11 \\
\hline 8 & 4.8 & 5.14 & 7.11 & 5.6 & 5.47 & 2.25 \\
\hline 9 & 7.9 & 8.54 & 8.09 & 6.9 & 7.80 & 13.04 \\
\hline 10 & 4.8 & 5.39 & 12.37 & 5.5 & 5.72 & 3.98 \\
\hline
\end{tabular}

\section{Acknowledgements}

The authors would like to thank Leaders of HaNoi University of Science and Technology, VietNam for providing financial support for project no. T2017-PC-110 titled "Research and Development of Noninvasive method measuring blood Glucose"

\section{References}

1 www.wpro.who.int/vietnam/mediacentre/ features/feature_world_health_day_2016_vietn am

2 So CF, Choi KS, Wong TK, Chung JW. Recent advances in noninvasive glucose monitoring. Med Devices (Auckl) 2012; 5: 45-52 DOI: 10.2147/MDER.S28134

3 Vonlilienfeldtoal H, Weidenmuller M, Xhelaj A, Mantele W, "A novel approach to noninvasive glucose measurement by mid-infrared spectroscopy: the combination of quantum cascade lasers (QCL) and photoacoustic detection". Vib Spectrosc. 2005;38(1-2), pp: 209-215.

4 Heise HM, Bittner A, Marbach R, "Clinical chemistry and near infrared spectroscopy: technology for non-invasive glucose monitoring". J Near Infrared Spec. 1998;6(4), pp:349-359.

5 Heise HM, Marbach R. Human oral mucosa studies with varying blood glucose concentration by non-invasive ATR-FT-IRspectroscopy. Cell Mol Biol (Noisy-le-grand) 1998; 44(6): 899-912 [PMID: 9763193]

6 Siesler HW, Ozaki Y, Kawata S, Heise HM, "Near-Infrared Spectroscopy: Principles, Instruments, Applications". Wiley-VCH Verlag GmbH; 2002. Pp: 1-348

7 Raghavachari R, " Near-Infrared Applications in Biotechnology". New York: Marcel Dekker; 2001, pp: 382 pages

8 Maruo $\mathrm{K}$, Tsurugi $\mathrm{M}$, Chin $\mathrm{J}$, et al. "Noninvasive blood glucose assay using a newly developed near-infrared system". IEEE J Sel Top Quant. 2003;9(2), pp: 322-330.

9 Arnold MA, Small GW. "Noninvasive glucose sensing". Anal Chem. 2005;77(17), pp: 54295439.

10 Berger AJ, Koo TW, Itzkan I, Horowitz G, Feld MS. Multicomponent blood analysis by nearinfrared Raman spectroscopy. Appl Opt 1999; 38(13): 2916-2926 [PMID: 18319874]

11 Hanlon EB, Manoharan R, Koo TW, Shafer KE, Motz JT, Fitzmaurice M, Kramer JR, Itzkan I, 
Dasari RR, Feld MS. Prospects for in vivo Raman spectroscopy. Phys Med Biol 2000; 45(2): R1-59 [PMID: 10701500]

12 Lee S, Nayak V, Dodds J, Pishko M, Smith NB. Glucose measurements with sensors and ultrasound. Ultrasound Med Biol 2005; 31(7): 971-977 [PMID: 15972203 DOI: 10.1016/j.ultrasmedbio.2005.04.004]
13 MacKenzie HA, Ashton HS, Spiers S, Shen Y, Freeborn SS, Hannigan J, Lindberg J, Rae P. Advances in photoacoustic noninvasive glucose testing. Clin Chem 1999; 45(9): 1587 1595 [PMID: 10471673]

14 Brince Paul, Melvin P Manuel, Zachariah C Alex . "Design and Development of Noninvasive Glucose Measurement System". MEMS and Sensors Division, School of Electronics Engineering VIT University, Vellore. Pp: 1-4. 